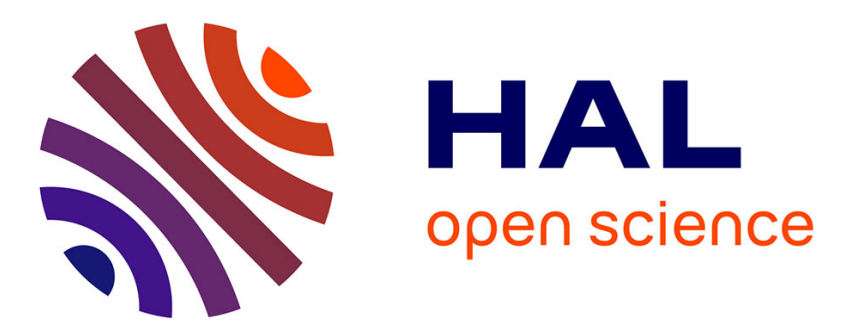

\title{
Directed self-avoiding walks on a randomly dilute lattice
}

\author{
J.P. Nadal, J. Vannimenus
}

\section{To cite this version:}

J.P. Nadal, J. Vannimenus. Directed self-avoiding walks on a randomly dilute lattice. Journal de Physique, 1985, 46 (1), pp.17-26. 10.1051/jphys:0198500460101700 • jpa-00209941

\section{HAL Id: jpa-00209941 https://hal.science/jpa-00209941}

Submitted on 1 Jan 1985

HAL is a multi-disciplinary open access archive for the deposit and dissemination of scientific research documents, whether they are published or not. The documents may come from teaching and research institutions in France or abroad, or from public or private research centers.
L'archive ouverte pluridisciplinaire HAL, est destinée au dépôt et à la diffusion de documents scientifiques de niveau recherche, publiés ou non, émanant des établissements d'enseignement et de recherche français ou étrangers, des laboratoires publics ou privés. 


\title{
Directed self-avoiding walks on a randomly dilute lattice
}

\author{
J. P. Nadal and J. Vannimenus \\ Groupe de Physique des Solides de l'Ecole Normale Supérieure, 24, rue Lhomond, 75231 Paris Cedex 05, France
}

(Reçu le 31 juillet 1984, accepté le 20 septembre 1984)

\begin{abstract}
Résumé. - Nous étudions un modèle de Marches sans retour Dirigées sur un réseau dilué, par différentes approches (arbre de Cayley, développement de faible désordre, calcul Monte-Carlo avec construction de marches jusqu'à 2000 pas). Ce modèle simple a l'avantage de présenter les traits essentiels du problème controversé des marches sans retour en milieu aléatoire. On montre en particulier que quelle que soit la valeur du désordre, valeur moyenne et valeur plus probable du nombre de marches sont différentes.
\end{abstract}

\begin{abstract}
We consider a model of Directed Self-Avoiding Walks (DSAW) on a dilute lattice, using various approaches (Cayley Tree, weak-disorder expansion, Monte-Carlo generation of walks up to 2000 steps). This simple model appears to contain the essential features of the controversial problem of self-avoiding walks in a random medium. It is shown in particular that with any amount of disorder the mean value for the number of DSAW is different from its most probable value.
\end{abstract}

\section{Introduction.}

The problem of Self-Avoiding Walks (SAW) on a randomly dilute lattice has been studied a lot recently [1-12], leading to conflicting conclusions. According to some authors [1,2], the statistical properties are the same as for the pure system, apart from a trivial renormalization of the connective constant; others $[4,5]$ have obtained similar conclusions, except that they give arguments for a change in the correlation length exponent $v$ at, and only at, the percolation threshold $p_{c}$. Rammal et al. [6] give arguments for a change (at least) at $p_{\mathrm{c}}$ of the exponent $v$. Numerical calculations [7] lead to a change in $v$ at any value of $p<1$. The latter result is in agreement with the original Harris criterion [8] for disordered systems. Also Derrida $[9,10]$ has argued that a change in all the statistics of the SAW should occur for any amount of disorder, and in particular, one should observe a difference between the mean value and the most probable value of the number of SAW of a given number of steps. In the first simulations on this subject Hiley et al. [11] did not observe such an effect, in agreement with authors quoted above [1-4]. On the contrary, very recently this effect was observed in 2-d Monte Carlo calculations by Roy and Chakrabarti [12]. Their work (exact enumeration of rather short SAW) also supports a charge in the exponent $\gamma$ for any value of $p$.
The purpose of this paper is to illustrate the arguments given in $[9,10]$ by considering a simpler model, namely the problem of Directed-Self-Avoiding Walks (DSAW) on a dilute lattice, for which accurate calculations can be done. In section 2 we recall the ideas of references $[9,10]$, and apply them to directed systems. In section 3 we discuss the general behaviour of the main quantities of interest for the DSAW model on a dilute lattice, and study this model on the Cayley Tree. In section 4 we study the model in two dimensions, first by a weak-disorder expansion, then on a strip of width 2 , and finally by Monte-Carlo calculations, using exact recursion relations to enumerate DSAW up to 2000 steps on random samples.

The main result is the difference obtained at any $p$, $0<p<1$, between the mean value and the most probable value of the number of DSAW of $N$ steps for $N$ large. We also briefly discuss the possibility of a change in the exponent $\gamma$ for any $p$.

\section{Self-avoiding walks on a dilute lattice.}

We briefly recall the arguments of references $[9,10]$. For the problem of SAW on a pure lattice, one quantity of interest is the number $\Omega_{N}$ of SAW of $N$ steps starting at a given point 0 . For large $N$, one expects [13]

$$
\Omega_{N} \sim \mu^{N} N^{\nu-1} .
$$


On a dilute lattice, where bonds are present with probability $p$, one has to consider for each configuration $\mathrm{C}$ of the lattice and for a given origin $\mathrm{O}$ the number $\omega_{N}(\mathrm{O}, \mathrm{C})$ of SAW of $N$ steps. It is easy to take the average over disorder ( $O$ being fixed) :

$$
\bar{\omega}_{N} \equiv \overline{\omega_{N}(\mathrm{O}, \mathrm{C})}=p^{N} \Omega_{N} \text {. }
$$

Relying on (2) it has been claimed [4] that $\gamma$ is not changed and that the sole effect of disorder is to change the connective constant $\mu$ into $p \mu$. However (2) shows that for $\mu^{-1}<p<p_{\mathrm{c}}, \bar{\omega}_{N}$ increases exponentially with $N$ while the lattice does not percolate, so that with probability one there is no $N$-step walk for $N$ very large. This is due to the fact that a few events of extremely small probability dominate the average, so that the most probable value $\omega_{N}^{*}$ of $\omega_{N}(\mathbf{O}, \mathbf{C})$ is different from its average even in the large $N$ limit :

$$
\omega_{N}^{*} \neq \bar{\omega}_{N} .
$$

Another argument in favour of a change in the statistics in presence of disorder is the general Harris criterion [8] for disordered systems, which says that if the exponent $\alpha$ (of the pure system) is positive, then any amount of disorder should change the statistics. In particular $\alpha$ is positive for SAW in any dimension $d$.

The preceding considerations apply as well for a directed system. Hence for example relations like (1), (2), (3) hold for the number of DSAW. In the pure case, the problem of DSAW on a regular lattice is a simple model recently studied [14]. Consider for example the hypercubic lattice in $d$ dimensions, with the preferred direction along the main diagonal. Then DSAW are walks starting at a given point $O$ and making steps only in one of the $d$ directions which have a positive projection along the preferred direction. As a result, no loops are possible, and the number of such walks with $N$ steps is simply

$$
\Omega_{N}=d^{N} .
$$

Each step has a projection $1 / \sqrt{d}$ along the preferred direction, therefore the mean end to end distance along that direction is

$$
\left\langle R_{\|}\right\rangle=L / \sqrt{d} .
$$

The DSAW reduce to a random walk orthogonally to the preferred direction, so that

$$
\left\langle R_{\perp}^{2}\right\rangle \sim L
$$

This says that in any dimension $d$,

$$
\begin{aligned}
& \mu=d, \\
& \gamma=v_{\|}=2 v_{\perp}=1 .
\end{aligned}
$$

These results are the same on a directed Cayley Tree of connectivity $z=1+d$ (the root $\mathrm{O}$ having only $d$ neighbours).
If we now consider DSAW on a dilute lattice, where each bond is present with probability $p$, we have to look at the number $\Omega_{N}(\mathrm{O}, \mathrm{C})$ of DSAW of $N$ steps starting at $\mathrm{O}$, for each configuration $\mathrm{C}$ of the lattice. Again it is easy to compute the average value $\bar{\omega}_{N}$ of $\omega_{N}(\mathrm{O}, \mathrm{C})$ :

$$
\bar{\omega}_{N}=p^{N} \Omega_{N}=(p d)^{N} .
$$

And again one can expect that the most probable value will be different from this mean value. Indeed, if $p_{\mathrm{c}}$ is now the threshold of directed percolation, for $1 / d<p<p_{c}, \bar{\omega}_{N}$ increases exponentially with $N$, while the probability that there is no $N$-step directed walk converges to 1 for very large $N$.

The length along the preferred direction of a $N$-step DSAW being the same in the dilute case as in the pure case, $v_{\|}$is still equal to 1 . Note however that $\left\langle R_{\|}\right\rangle$- hence also $v_{\|}$- is defined only for clusters C where $\omega_{N}(\mathbf{O}, \mathbf{C})$ is not zero, while for example (7) is obtained by averaging over all the clusters.

The Harris criterion can also easily be generalized to directed systems. One can follow the argument of Harris [8], taking into account two typical lengths $\xi_{\|}$and $\xi_{\perp}$ instead of one, one along and one orthogonally to the preferred direction, associated with two different exponents $v_{\|}$and $v_{\perp}$. Then one finally obtains the criterion :

if $v_{\|}+(d-1) v_{\perp}-2<0$, then disorder should be relevant .

For systems where the hyperscaling relation $(d-1) v_{\perp}+v_{\|}=2-\alpha$ holds, (8) reads the same as for non directed systems.

If we apply this criterion to Directed Animals on a dilute lattice, we find that disorder should be relevant for $d<d_{\mathrm{c}}=7$. The DSAW problem seems particular in that for any $d, v_{\|}=2 v_{\perp}=1$. If we apply (8), then critical exponents should be changed by disorder for $d<3$.

In the next section we consider some simple properties of the distribution of DSAW on a dilute lattice.

\section{Directed SAW on a dilute lattice.}

We now consider the model introduced in the previous section, i.e. DSAW on the hypercubic lattice in $d$ dimensions, where each bond is present with probability $p$, and absent with probability $(1-p)$ and the preferred direction is along the main diagonal.

We have to consider the distribution $D_{N}(\omega)$ for the number of DSAW of $N$ steps starting at a given origin O. One can write

$$
D_{N}(\omega)=\left(1-P_{N}\right) \delta(\omega)+P_{N} \Delta_{N}(\omega),
$$

where $P_{N}$ is the probability that there exists at least one directed path of $N$ steps, and $\Delta_{N}(\omega)$ is the (normalized) distribution for the number $\omega_{N}$ of DSAW of 
$N$ steps on clusters extending at least up to $N$. When $N$ goes to infinity, $P_{N}$ converges toward $P_{\infty}(p)$, the probability that the origin belongs to the infinite cluster :

$$
\lim _{N \rightarrow \infty} P_{N}=P_{\infty}(p)
$$

Clearly, for $p<p_{c}$, the most probable value for $\omega_{N}$ is zero, in the large $N$ limit. At any $p$ or $N$, with probability $P_{N}(p), \omega_{N}$ is not zero and is distributed according to $\Delta_{N}$. In particular in this case one is interested in the behaviour of the most probable value $\omega_{N}^{*}$, which gives the growth constant $A^{*}(p)$ :

$$
\lim _{N \rightarrow \infty} \frac{1}{N} \log \omega_{N}^{*}=A^{*}(p) .
$$

We now define several averaged quantities. We will show that for $0<p<1$ they do not have the same behaviour in the large $N$ limit, and will give some arguments to identify the growth constant $A^{*}(p)$ with one of them. We have already defined the mean value of $\omega$ over the full distribution :

$$
\bar{\omega}_{N}=\sum_{\omega} D_{N}(\omega) \omega=(d p)^{N} .
$$

Similarly, we consider the mean value over $\Delta_{N}$ :

$$
\tilde{\omega}_{N}=\sum_{\omega} \Delta_{N}(\omega) \omega=\frac{1}{P_{N}}(d p)^{N}
$$

Now consider the following quantities :

$$
a_{N}(p)=\frac{1}{N} \log \left(1+\bar{\omega}_{N}\right)
$$

$$
\begin{aligned}
& b_{N}(p)=\frac{1}{N} \log \left(1+\tilde{\omega}_{N}\right), \\
& A_{N}(p)=\frac{1}{N} \sum_{\omega} D_{N}(\omega) \log (1+\omega), \\
& B_{N}(p)=\frac{1}{N} \sum_{\omega} \Delta_{N}(\omega) \log (1+\omega) .
\end{aligned}
$$

and the corresponding quantities obtained in the infinite $N$ limit :

$$
a(p), b(p), A(p), B(p)
$$

(the additive constant 1 in the logarithms has been introduced to avoid divergences in $\left.A_{N}(p)\right)$. All these four numbers take the common value $\log d$ at $p=1$. Also, one has :

$$
\begin{aligned}
a(p) & =\log (d p), & & p>1 / d, \\
& =0, & & p<1 / d,
\end{aligned}
$$

and the following general relations

$$
\begin{gathered}
b_{N}(p)=\log (d p)-\frac{1}{N} \log P_{N}, \\
A_{N}(p)=P_{N} B_{N}(p) .
\end{gathered}
$$

Due to the convexity of the logarithm function, one has for any $p$ and any $N$ :

$$
A_{N}(p) \leqslant a_{N}(p), \quad B_{N}(p) \leqslant b_{N}(p) .
$$

In the limit $N \rightarrow \infty$, (16) gives

$$
A(p)=P_{\infty}(p) B(p),
$$

and (15) gives, for $N$ going to infinity :

$$
\begin{array}{ll}
p>p_{\mathrm{c}}: P_{N} \sim P_{\infty}(p), & b(p)=a(p), \\
p=p_{\mathrm{c}}: P_{N} \sim N^{-\beta / v_{\|},} & b(p)=a(p), \\
p<p_{\mathrm{c}}: P_{N} \sim \exp \left[-N / \xi_{\|}(p)\right], & b(p)=a(p)+\frac{1}{\xi_{\|}(p)},
\end{array}
$$

(where $\xi_{\|}(p)$ is the correlation length along the preferred direction).

On these relations one can deduce some simple properties, which are summarized [15] on figure 1 :

i) If $\Delta_{N}$ were peaked around its mean value $\tilde{\omega}_{N}$, one would have, for $p>p_{c}, B(p)=b(p)=a(p)=$ $\log (d p)$. This has led several authors to identify the most probable value $A^{*}(p)$ with $a(p)$, but this identification is not justified if these quantities are not equal.

ii) Since $B$ and $A$ are finite quantities, bounded by their common value $\log d$ at $p=1$, one sees from
(18) that $A(p)$ is singular at $p_{\mathrm{c}}$ - whereas $a(p)$ is not. In particular

$$
A(p)=0 \text { for } p<p_{\mathrm{c}} .
$$

Again this is not surprising since for $p<p_{\mathrm{c}}$ the most probable value for the full distribution of $\omega_{N}$ in the large $N$ limit must be 0 .

iii) Let us now look at the quantities obtained from $\Delta_{N}$. Knowing the behaviour of $\xi_{\|}$near $p_{\mathrm{c}}[18]$ :

$$
\xi_{\|}(p) \sim\left|p-p_{\mathrm{c}}\right|^{-v(p)},
$$




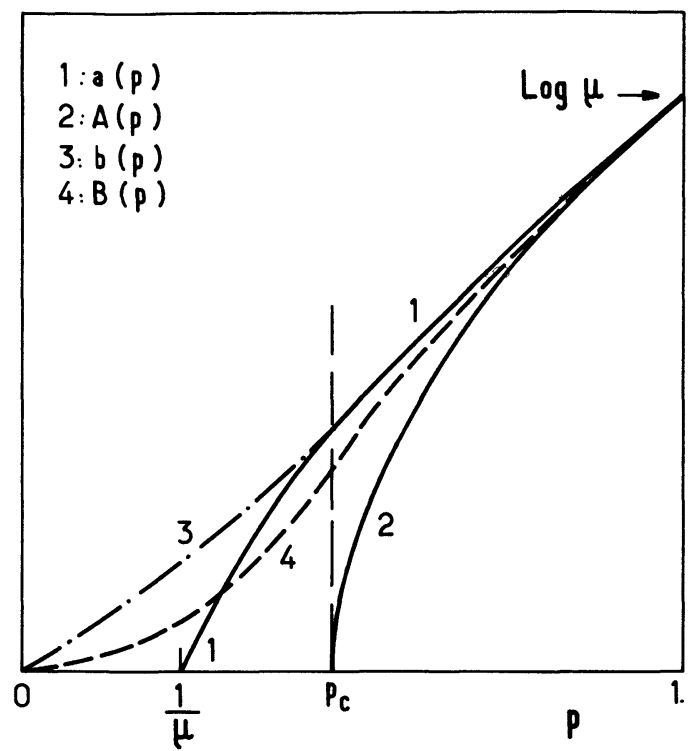

Fig. 1. - Qualitative behaviour of the averaged quantities $a$, $b, A, B$, defined in the text, as functions of $p$. In particular, $A(p)$ is singular at $p_{\mathrm{c}}, a(p)$ vanishes at $p=\mu^{-1}=d^{-1}$, $b(p)$ and $B(p)$ have an inflexion point at $p_{\mathrm{c}}$.

with $v_{\|}^{(p)}>1$ in all $d<5$, equation (19) implies that $b(p)$, has an inflexion point at $p_{c}$. According to our numerical calculations in 2 dimensions described here after, it seems likely that $B$ has a qualitative behaviour similar to $b$, but with $B(p)<b(p)$ for any $p$ in $] 0,1[$.

iv) In the limit $p \rightarrow 0$, since $P_{N}$ behaves like $(d p)^{N}$, one has

$$
\lim _{p \rightarrow 0} \tilde{\omega}_{N}=1
$$

thus

$$
\lim _{p \rightarrow 0} b_{N}=\lim _{p \rightarrow 0} b=0,
$$

and using (17)

$$
\lim _{p \rightarrow 0} B_{N}=\lim _{p \rightarrow 0} B=0 .
$$

Note that this gives the behaviour of $\xi_{\|}(p)$ in this limit, from (19) :

$$
\lim _{p \rightarrow 0}\left(\frac{1}{\xi_{\|}(p)}+\log p\right)=-\log d .
$$

We also note here that in the preceding considerations no restriction is put on the size of the clusters : another problem which might be interesting in the study of (directed) percolation clusters would be the study of (D) SAW on clusters of a given number of sites S. Other statistics would be obtained, and in particular in the limit $p \rightarrow 0$, one would find the statistics of (D) SAW on (directed) animals of a given size $S$.
We will argue below that the most probable growth constant is given by $B(p)$, and not by $b(p)$ or $a(p)$. Before that, we now consider various analytical approaches to the DSAW model, on a dilute Cayley Tree, on strips and by a weak-disorder expansion.

\section{Analytical approaches.}

4.1 Results on the Cayley tree. - We consider a Directed Cayley Tree (DCT) defined as a Cayley tree of connectivity $z=1+\sigma$, with an origin $\mathrm{O}$ having only $\sigma$ next nearest neighbours. Note that if we consider the DCT locally embedded in a cubic lattice, $\sigma$ plays the role of a dimension. We consider the site model : each site is present with probability $p$. The number of DSAW of $N$ steps starting from $O$ on the dilute DCT is identical to the number of sites that are accessible in exactly $N$ steps from $\mathrm{O}$ on the dilute DCT, a quantity recently studied [16], which is related to the "spreading dimension " of the percolation cluster. This identity is due to the absence of loops on a Cayley tree. As a result, at $p_{\mathrm{c}}$, the mean number of DSAW $\bar{\omega}_{N}$ is a constant when the average is performed over all possible configurations :

$$
\bar{\omega}_{N}=\frac{1}{\sigma}
$$

whereas if one only considers walks on clusters extending at least up to $N$, the mean number $\widetilde{\omega}_{N}$ of these walks [16] grows like $N$ (for $N$ large, at $p_{\mathrm{c}}$ )

$$
\widetilde{\omega}_{N} \sim \frac{\sigma-1}{2 \sigma} N .
$$

Here we are interested in the fluctuations of $\omega_{N}$ in both cases, and not only at $p_{\mathrm{c}}$.

On the DCT, the (directed) percblation threshold is

$$
p_{\mathrm{c}}=1 / \sigma
$$

and the number of DSAW of $N$ steps (for the pure case) :

$$
\Omega_{N}=\sigma^{N-1},
$$

so that the growth constant $\mu=\sigma$ is equal to $p_{\mathrm{c}}^{-1}$. We see on figure 1 that in this case all the curves will be identical, at least for $p \leqslant p_{c}$. However, even in this case the fluctuations can be very large. To see this, one can consider the distribution $D_{N}(\omega)$ for the number of DSAW of $N$ steps on the dilute DCT.

One obtains recursion relations for its generating function $U_{N}(z)$ :

$$
\begin{aligned}
& U_{N}(z) \equiv \overline{\exp z \omega_{N}}=\sum_{\omega} D_{N}(\omega) \exp (z \omega), \\
& U_{N}(z)=1-p+p\left[U_{N-1}(z)\right]^{\sigma}, \\
& U_{1}(z)=1-p+p \mathrm{e}^{z} .
\end{aligned}
$$


Any moment of $\omega_{N}$ can be obtained from (25), as for example

$$
\bar{\omega}_{N}=\frac{1}{\sigma}(p \sigma)^{N}=p^{N} \Omega_{N} .
$$

One can also deduce from (25) the probability $P_{N}$ for $\omega_{N}$ to be non zero :

$$
P_{N+1}=p\left(1-\left(1-P_{N}\right)^{\sigma}\right), \quad P_{1}=p,
$$

and thus one obtains $P_{\infty}$ as a fixed point of (27) $\left(P_{\infty}=0\right.$ for $p \leqslant p_{\mathrm{c}}=\frac{1}{\sigma}, P_{\infty}>0$ for $\left.p>p_{\mathrm{c}}\right)$, and the moments of $\omega_{N}$ according to $\Delta_{N}$ :

$$
\widetilde{\omega_{N}^{n}} \equiv \sum_{\omega} \Delta_{N}(\omega) \omega^{n}=\overline{\omega_{N}^{n}} / P_{N} .
$$

The behaviour of $P_{N}$ for large $N$ is the following :

$$
\begin{gathered}
p>p_{\mathrm{c}}: P_{N} \sim P_{\infty} ; \quad p=p_{\mathrm{c}}: P_{N} \sim \frac{2}{(\sigma-1) N} ; \\
p<p_{\mathrm{c}}: P_{N} \sim(p \sigma)^{N} .
\end{gathered}
$$

For the relative fluctuations

$$
S_{N} \equiv \frac{\overline{\omega_{N}^{2}}-\bar{\omega}_{N}^{2}}{\bar{\omega}_{N}^{2}}, \quad \Sigma_{N} \equiv \frac{\widetilde{\omega_{N}^{2}}-\widetilde{\omega}_{N}^{2}}{\widetilde{\omega}_{N}^{2}},
$$

one obtains in the large $N$ limit :

$$
\begin{aligned}
p>p_{\mathrm{c}} \lim _{N \rightarrow \infty} S_{N}= & \frac{\sigma(1-p)}{p \sigma-1}, \\
& \lim _{N \rightarrow \infty} \Sigma_{N}=P_{\infty} \frac{\sigma-1}{p \sigma-1}-1,
\end{aligned}
$$

$$
p=p_{\mathrm{c}}: S_{N} \sim N(\sigma-1), \lim _{N \rightarrow \infty} \Sigma_{N}=1 .
$$

One can also see that at $p_{\mathrm{c}},\left(\omega_{N}\right)^{n}$ does not depend on $N$, $\overline{\omega_{N}^{n}}$ is a polynomial in $N$ of degree $n-1$, and $\widetilde{\omega}_{N}^{h}$ of degree $n$. Thus we see that at $p_{c}$, even though all the quantities give the same growth constant, the fluctuations of $\omega_{N}$ are very large.

4.2 WEAK-DISORDER EXPANSION IN 2 DIMENSIONS. Consider the square lattice with the preferred direction along the diagonal. At the lowest order in $q$ (probability for a site to be empty), we look, at the probability distribution $D_{N}(\omega)$ of the number $\omega$ of DSAW of $N$ steps starting at a given occupied site (Fig. 2).

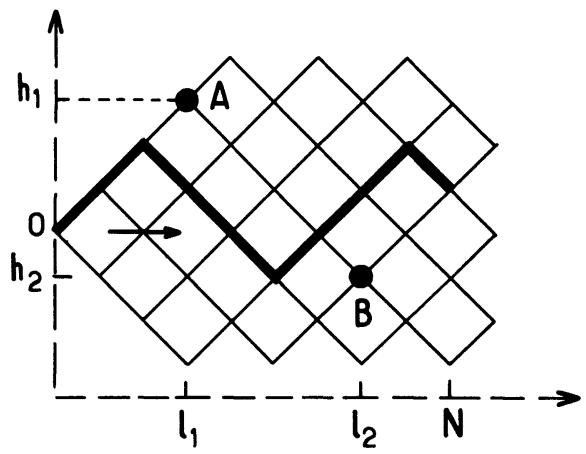

Fig. 2. - A directed self-avoiding walk.(DSAW) on the dilute square lattice (heavy line). The preferred direction, along the main diagonal, is indicated by the arrow. All sites are present, except the two sites $\mathbf{A}$ and $\mathbf{B}$ of coordinate $\left(l_{1}, h_{1}\right)$ and $\left(l_{2}, h_{2}\right)$.

At order $q^{2}$, one gets

$$
\begin{aligned}
D_{N}(\omega)=\delta\left(\omega-2^{N}\right) & +q \sum_{\mathbf{A}}^{\prime}\left[\delta\left(\omega-\Omega_{N}^{(1)}(\mathrm{A})\right)-\delta\left(\omega-2^{N}\right)\right]+ \\
& +q^{2} \sum_{(\mathbf{A}, \mathbf{B})}^{\prime}\left[\delta\left(\omega-\Omega_{N}^{(2)}(\mathrm{A}, \mathrm{B})\right)-\delta\left(\omega-\Omega_{N}^{(1)}(\mathrm{A})\right)-\delta\left(\omega-\Omega_{N}^{(1)}(\mathrm{B})\right)+\delta\left(\omega-2^{N}\right)\right],
\end{aligned}
$$

where $\Omega_{N}^{(1)}(\mathrm{A})\left(\right.$ resp. $\left.\Omega_{N}^{(2)}(\mathrm{A}, \mathrm{B})\right)$ is the number of DSAW when the site $\mathrm{A}$ is missing, (resp. when A and B are missing), and the primed sum $\sum_{(\mathbf{A}, \mathbf{B})}^{\prime}$ is over each pair of different sites (A, B) - also different from $O$. Each point is characterized by the distance $l$ from the source along the main axis (preferred direction) and the height $h$ orthogonally to it (Fig. 2). Denoting by $g(l, h)$ the fraction of walks reaching point $(l, h)$ (on the non dilute lattice), one has

$$
g(l, h)=C_{l}^{(l+h) / 2} 2^{-l}
$$

and one obtains for $\mathrm{A}=(l, h), 1 \leqslant l \leqslant N$

$$
\Omega_{N}^{(1)}(\mathrm{A})=2^{N}(1-g(l, h)),
$$

and for $\mathrm{A}=\left(l_{1}, h_{1}\right), \mathrm{B}=\left(l_{2}, h_{2}\right), 1 \leqslant l_{1} \leqslant l_{2} \leqslant N$ :

$$
\Omega_{N}^{(2)}(\mathrm{A}, \mathrm{B})=2^{N}\left(1-g\left(l_{1}, h_{1}\right)-g\left(l_{2}, h_{2}\right)+g_{12}\right),
$$


with

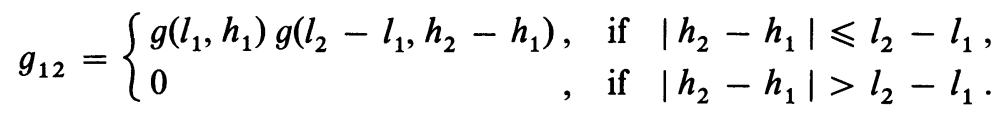

As $\bar{\omega}_{N}=p^{N} 2^{N}$, at second order in $q$

$$
\frac{1}{N} \log \bar{\omega}_{N}=\log 2-q-\frac{q^{2}}{2},
$$

and one can check that this result is also obtained from(31). Now, from(31)

$$
\frac{1}{N} \overline{\log \omega_{N}}=\log 2+q \frac{1}{N} \sum_{l=1}^{N} \sum_{m=0}^{l} \log \left(1-C_{l}^{m} 2^{-l}\right)+q^{2}\left[\frac{1}{N} \sum_{\mathbf{A}, \mathbf{B}}^{\prime} \log \frac{\Omega_{N}^{(2)}(\mathbf{A}, \mathbf{B}) 2^{N}}{\Omega_{N}^{(1)}(\mathrm{A}) \Omega_{N}^{(1)}(\mathbf{B})}\right] .
$$

As $N$ goes to infinity, the term of order $q$ is

$$
-q\left(1+0\left(\frac{1}{\sqrt{N}}\right)\right)
$$

and thus is identical in the thermodynamic limit with the corresponding term in $\log \bar{\omega}_{N}$, whereas the term of order $q^{2}$ is

$$
-q^{2}\left(\frac{1}{2}+\varepsilon\right)
$$

with $\varepsilon \sim 0.16$ obtained numerically.

This shows that with any amount of disorder the logarithm of the mean value and the mean value of the logarithm are different.

4.3 DSAW ON A STRIP. - Let us illustrate the preceding considerations on the simple case of site DSAW on a dilute strip of width 2 , drawn on the directed square lattice, with periodic boundary conditions : on every column, there are two sites, each of them being neighbour of each site of the previous column. If $\omega_{N}(i)$ is the number of DSAW of $N$ steps arriving at site $i$ on the $N$ th column, we have the recursion relation

$$
\left(\begin{array}{l}
\omega_{N}(1) \\
\omega_{N}(2)
\end{array}\right)=\left(\begin{array}{l}
\alpha_{1}(N) \alpha_{1}(N) \\
\alpha_{2}(N) \alpha_{2}(N)
\end{array}\right)\left(\begin{array}{l}
\omega_{N-1}(1) \\
\omega_{N-1}(2)
\end{array}\right)
$$

where $\alpha_{i}(N)$ is 1 if site $i$ is present on the column $N$, and 0 otherwise. Then $\omega_{N}$ is given by a product of random matrices :

$$
\left(\begin{array}{l}
\omega_{N}(1) \\
\omega_{N}(2)
\end{array}\right)=M_{N} M_{N-1} \ldots M_{1}\left(\begin{array}{l}
\omega_{0}(1) \\
\omega_{0}(2)
\end{array}\right) .
$$

The total number $\omega_{N}$ of DSAW is the sum $\omega_{N}(1)+\omega_{N}(2)$, and in this particular example $\omega_{N}$ obeys a simple recursion relation

$$
\omega_{N}=\left(\alpha_{1}(N)+\alpha_{2}(N)\right) \omega_{N-1},
$$

so that one obtains

$$
\begin{array}{r}
D_{N}(\omega)=\left[1-\left(1-q^{2}\right)^{N}\right] \delta(\omega)+ \\
+\left(1-q^{2}\right)^{N} \Delta_{N}(\omega), \\
\Delta_{N}(\omega)=\int \prod_{j=1}^{N} \mathrm{~d} t_{j} \rho\left(t_{j}\right) \delta\left(\omega-\prod_{j=1}^{N} t_{j}\right)
\end{array}
$$

where $\rho(t)$ is the distribution probability of $t=\alpha_{1}+\alpha_{2}$ under the condition that $t$ is not 0 :

$$
\rho(t)=\frac{2(1-p)}{2-p} \delta(t-1)+\frac{p}{2-p} \delta(t-2) .
$$

In the present case where $\omega_{N}$ is given by a product of independent random variables, it is well known that the distribution $\Delta_{N}(\omega)$ is log-normal. The most probable value $\omega_{N}^{*}$ is then obtained from the mean value of the logarithm :

$$
\omega_{N}^{*}=\exp (N B(p))
$$

with

$$
B(p)=\int \rho(t) \log t \mathrm{~d} t=\frac{p}{2-p} \log 2,
$$

and not by $\exp (\mathrm{Na}(\mathrm{p}))$ :

$$
a(p)=\log \left[\int \rho(t) t \mathrm{~d} t\right]=\log \frac{2}{2-p} .
$$

For a strip of width $n \geqslant 3$ and for the 2-d systems, $\omega_{N}$ is still obtained from a product of independent random matrices, but the calculation of $\Delta_{N}$ does not reduce to a product of independent random variables. However it is reasonable to expect that the most probable value according to $\Delta_{N}$ is still given by $\exp \left(N B_{N}(p)\right), B_{N}(p)$ being the mean value of the logarithm. This is confirmed by the following MonteCarlo calculations. 


\section{Monte-Carlo calculation for the square lattice.}

5.1 MethOD. - The preferred direction is taken along the main diagonal of the square lattice. For a given dilute network, we enumerate exactly the number of DSAW starting from a given point, with a given number $N$ of steps. We then calculate the averaged quantities $a_{N}, A_{N}, B_{N}$, and also study the full distribution $D_{N}$ of $\omega_{N}$. This was done for $N$ up to 2000 , and various values of $p$, with particular attention to the neighbourhood of $p_{c}$. We consider both the site and the bond problems.

The exact enumeration can be obtained very simply (for a given network) because it is possible to write recursion relations between the numbers $\omega_{N}(h)$ of walks arriving at site $h$ on the $N$ th column orthogonal to the preferred direction. This enables us to study much longer walks than is possible for isotropic SAW models.

For the site model, one has

$\omega_{N}(h)=\alpha_{N}(h)\left[\omega_{N-1}(h-1)+\omega_{N-1}(h+1)\right]$,

where the random variable $\alpha_{N}(h)$ is the occupancy of site $(L, h)$, and takes the value 1 with probability $p$, and 0 with probability $(1-p)$. Similarly, for the bond problem :

$$
\begin{aligned}
\omega_{N}(h)=\alpha_{N}^{-}(h) \omega_{N-1}(h-1) & + \\
& +\alpha_{N}^{+}(h) \omega_{N-1}(h+1)
\end{aligned}
$$

where $\alpha_{N}^{ \pm}(h)$ are the random variables associated with occupancy of the bond between the sites $(N, h)$ and $(N-1, h \pm 1)$.

\section{Starting with}

$$
\omega_{0}(h)=\alpha_{0}(0) \delta_{h, 0},
$$

one can iterate (47) column after column. One sample is terminated when a column is encountered where no site (or bond) is found connected to the origin.

5.2 Numerical RESUlts. - On figure 3 we show for the site model the mean value $\bar{\omega}_{N}$ obtained on about 2500 samples, for $N<100$, and at different values of p. As shown above, $\frac{\log \bar{\omega}_{N}}{N}$ should be independent of $N$. Clearly, as $N$ grows and $p$ decreases, it becomes harder and harder to recover this result numerically : this is due to the fact that the mean value is dominated by very rare events. For $p<p_{c}$, the numerically obtained $\left(\log \bar{\omega}_{N}\right) / N$ stays near its theoretical value as long as $N$ is less than the typical length of the clusters.

On the contrary, one obtains more easily accurate values of $\log \left(\overline{\left.1+\omega_{N}\right)}\right.$ even for large $N$. On figure 4 $A_{N}(p)$ and $B_{N}(p)$ are shown as functions of $p$, together with $a(p)=\log (2 p)$. Clearly $A_{N}$ becomes singular

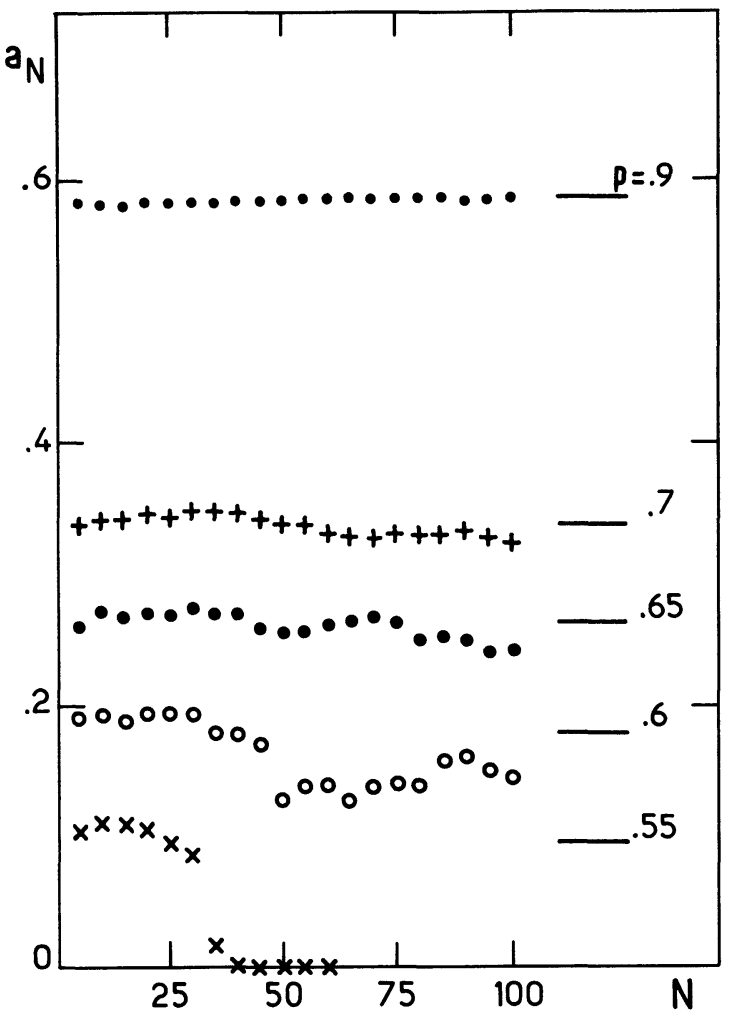

Fig. 3. - Numerical results for $N^{-1} \log \bar{\omega}_{N}$ versus the number of steps $N$, for different values of $p$, for the site dilution model : $\bar{\omega}_{N}$ is here the value obtained by averaging over about 2500 samples. One sees that for $p<p_{\mathrm{c}} \simeq 0.705$, $\frac{1}{N} \log \bar{\omega}_{N}$ drops for large $N$ below its theoretical value (Eq. (14)), indicated by a segment for each $p$.

at a value $p^{*}$ when $N$ goes to infinity. For the site problem we obtain

$$
p_{\mathrm{S}}^{*}=0.705 \pm 0.005,
$$

and for the bond problem

$$
p_{\mathbf{B}}^{*}=0.6445 \pm 0.0010,
$$

by analysing the size dependence of our results. These values agree well with known estimates [17-18] of the critical probabilities for the directed site and bond percolation problems on the square lattice : $p_{\mathbf{c}}^{(\mathbf{S})}=$ $0.7058 \pm 0.0005$ and $p_{\mathrm{c}}^{(\mathrm{B})}=0.6445 \pm 0.0005$. It seems likely from figure 4 that $B(p)$ has an inflexion point at $p_{\mathrm{c}^{-}}$.

We also checked that at $p_{\mathrm{c}}$ the probability $P_{L}$ behaves with a power law of $L$ in the range of length studied : $P_{L} \sim L^{-\beta / v_{\|}}$. Our results give a value of $\sim 0.16$ for the exponent, in good agreement with known values of $\beta$ and $v_{\|}$for directed percolation [18].

On figure 5 we show the probability distribution $\pi_{N}$ of $U=N^{-1} \log \left(1+\omega_{N}\right)$ for $N=10^{3}$ for the site model near the percolation threshold $(p=0.705) . \pi_{N}$ is peaked around its most probable value $B_{N}(p)$, and 


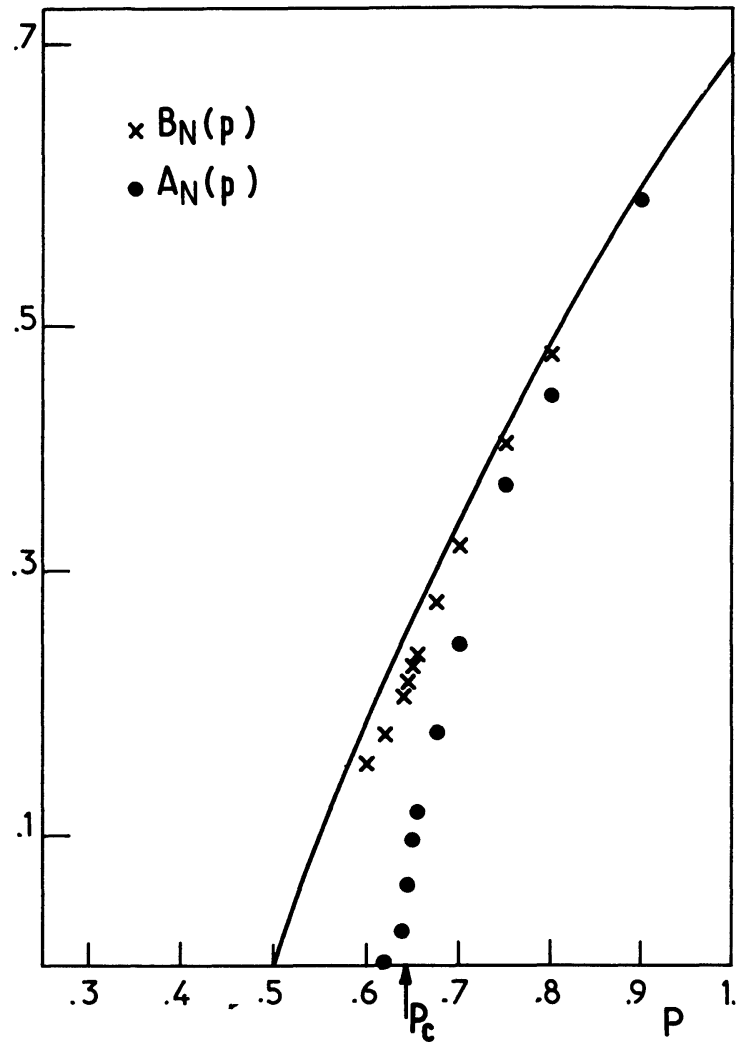

a)

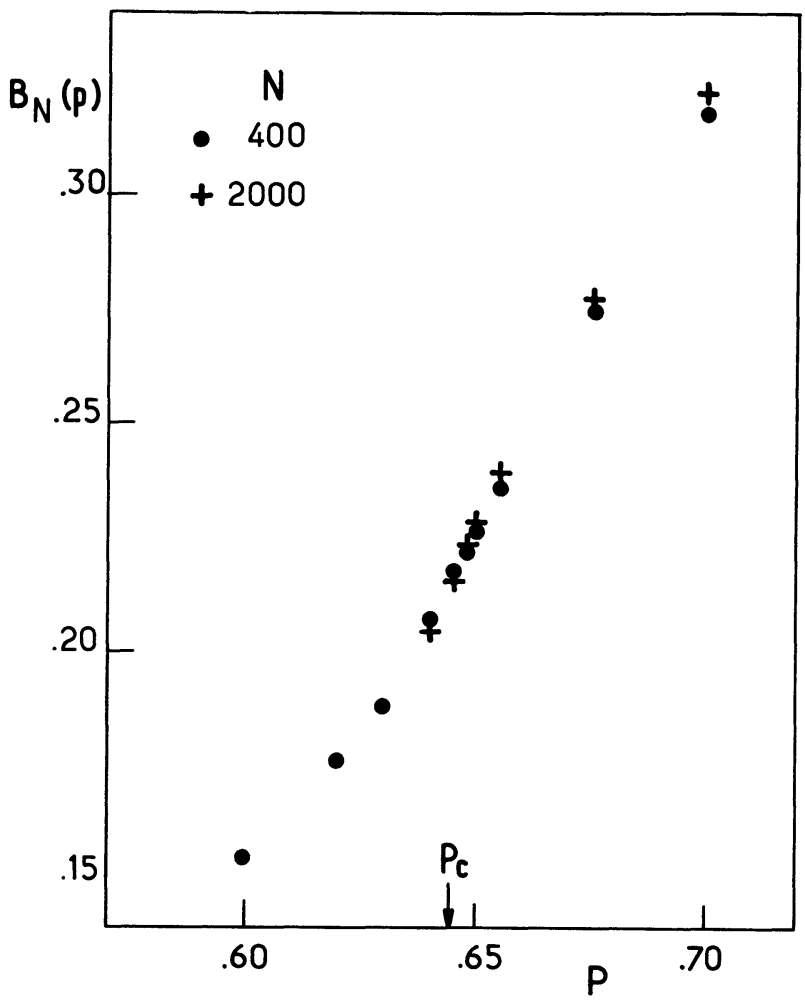

b)

Fig. 4. - a) Monte Carlo calculations in 2 dimensions for the bond dilution model. We show $A_{N}(p)=\frac{1}{N} \overline{\log \left(1+\omega_{N}\right)}$ and $B_{N}(p)=A_{N}(p) / P_{N}(p)$. for $N=2000$. The points lie below the solid curve $a(p)=\log 2 p$. At this scale, the points do not differ significantly from their asymptotic value $A(p)$ or $B(p)$. except for $A_{N}(p)$ very near $p_{\mathrm{c}}=0.6445$ (denoted by an arrow) : at $p_{\mathrm{c}} A_{N}(p)$ goes to zero as $N$ goes to infinity. b) Magnified portion of the curve $B_{N}(p)$ near $p_{\mathrm{c}}$. for $N=400$ and $N=2000$. It suggests that in the limit of infinite $N$ the inflection point of $B(p)$ will be at $p_{c}$.

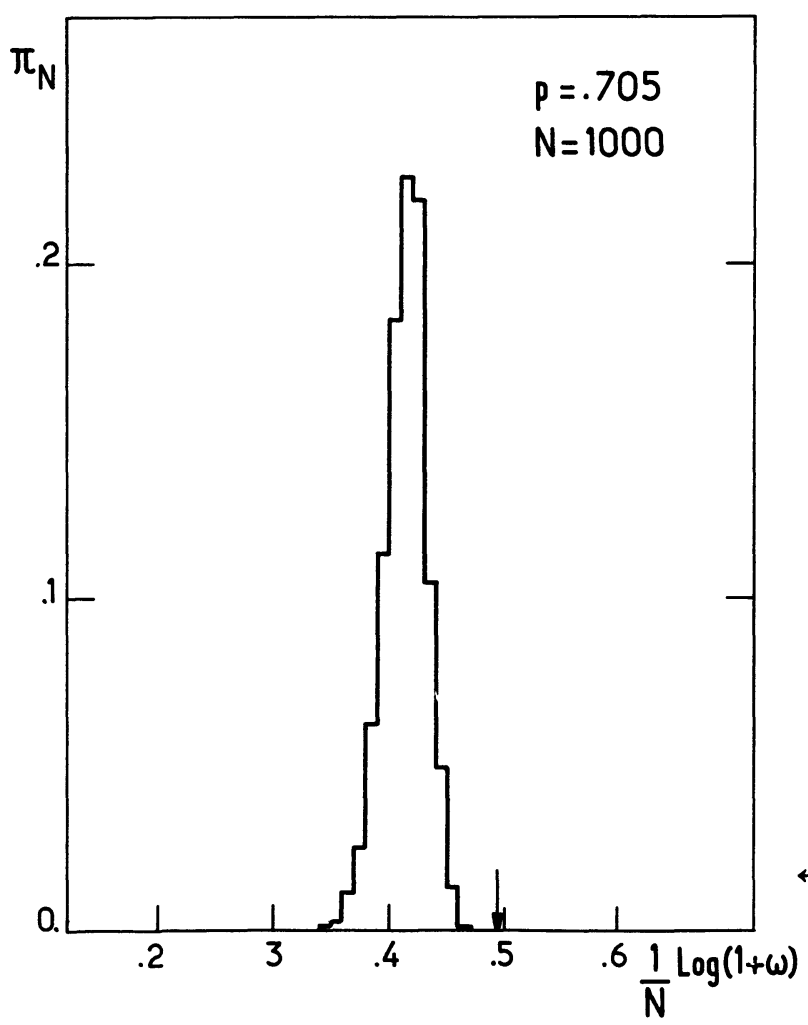

$a_{N}$ and $b_{N}$ lie outside this peak. This shows that the distribution has a long tail at large values of $\omega_{N}$, which is unobservable numerically.

The probability distribution of $U$ for the bond model at threshold is displayed in figure 6, for two values of $N$. One sees that the distributions closely coincide when scaled by factors $N^{1 / 2}$ : this shows that the width of $\pi_{N}$ vanishes like $N^{1 / 2}$ for large $N$ at $p_{\mathrm{c}}$, and suggests that it follows a scaling law. We also checked that such a scaling law exists for $p>p_{\mathrm{c}}$, but with another power of $N$.

5.3 EFFECTIVE EXPONENT $\gamma$. - As stated in the introduction, there is much debate on the influence of dilution on the critical exponents of SAW. By analogy with equation (1) for the pure system, one might

$\leftarrow$ Fig. 5. - Distribution $\pi_{N}$ of $u=\frac{1}{N} \log \left(1+\omega_{N}\right)$, for the site dilution model at $p \simeq p_{\mathrm{c}}$, for $N=1000$. The arrow indicates the value $a(p)=\log 2 p=0,495695 . .$. , which lies outside the peak of the distribution. 


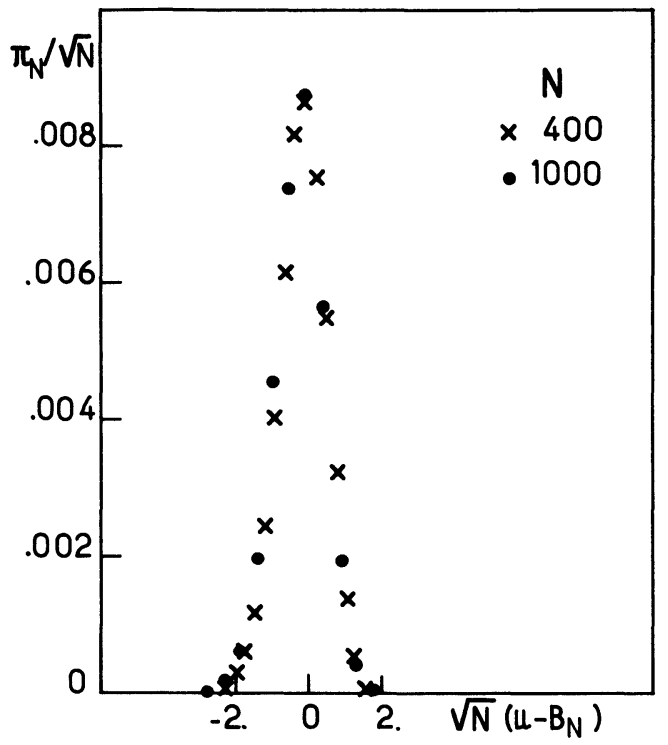

Fig. 6. - Distribution $\pi_{N}$ of $u=\frac{1}{N} \log \left(1+\omega_{N}\right)$, for the bond dilution model at $p \simeq p_{\mathrm{c}}=0.6445$, for $N=400$ and $N=10^{3}$. Here $\pi_{N} / \sqrt{N}$ is plotted as a function of $\sqrt{N}\left(u-B_{N}\right)-B_{N}$ being the mean value of $u$-, and the similarity of the two curves suggests the existence of a scaling law for $\pi_{N}$.

expect that the most probable value $\omega_{N}^{*}$ in the dilute system has an asymptotic expansion of the form

$$
\frac{1}{N} \log \omega_{N}^{*}-A^{*}(p) \sim(\gamma-1) \frac{\log N}{N},
$$

where $\gamma$ may now depend on $p$. However, one cannot a priori exclude that the expansion is more complicated and contains terms of order $N^{1 / 2}$ for example.

If expansion (49) is correct, one can expect a similar behaviour for $B_{N}(p)$ :

$$
B_{N}(p) \sim B(p)+(\gamma-1) N^{-1} \log N .
$$

In figure 7, $B_{N}(p)$ is shown as a function of $N^{-1} \log N$, for different values of $p$. Assuming that equation (50) holds for DSAW, we extract an effective exponent $\gamma(p)$ from the slope of the curves for $N>10^{3}$. This gives $\gamma<1$, for $p>p_{\mathrm{c}},(\gamma \simeq 0.70$ for $p=0.75)$, $\simeq 1$ for $p=p_{\mathrm{c}}$ and $>1$ for $p<p_{\mathrm{c}}$.

We also see on figure 7 that for each $p>p_{\mathrm{c}}$ there exists some $N(p)$ such that the slope is positive and small for $N<N(p)$ and crosses over to a different value for $N>N(p)$. A crossover has also been observed by Roy and Chakrabarty [12] for the nondirected case. This shows that it is necessary to study long walks, but even in the directed model where we are able to study much longer walks than in other situations, it is not possible to distinguish numerically between corrections in $N^{-1} \log N$ and, say, $N^{-1 / 2}$ :

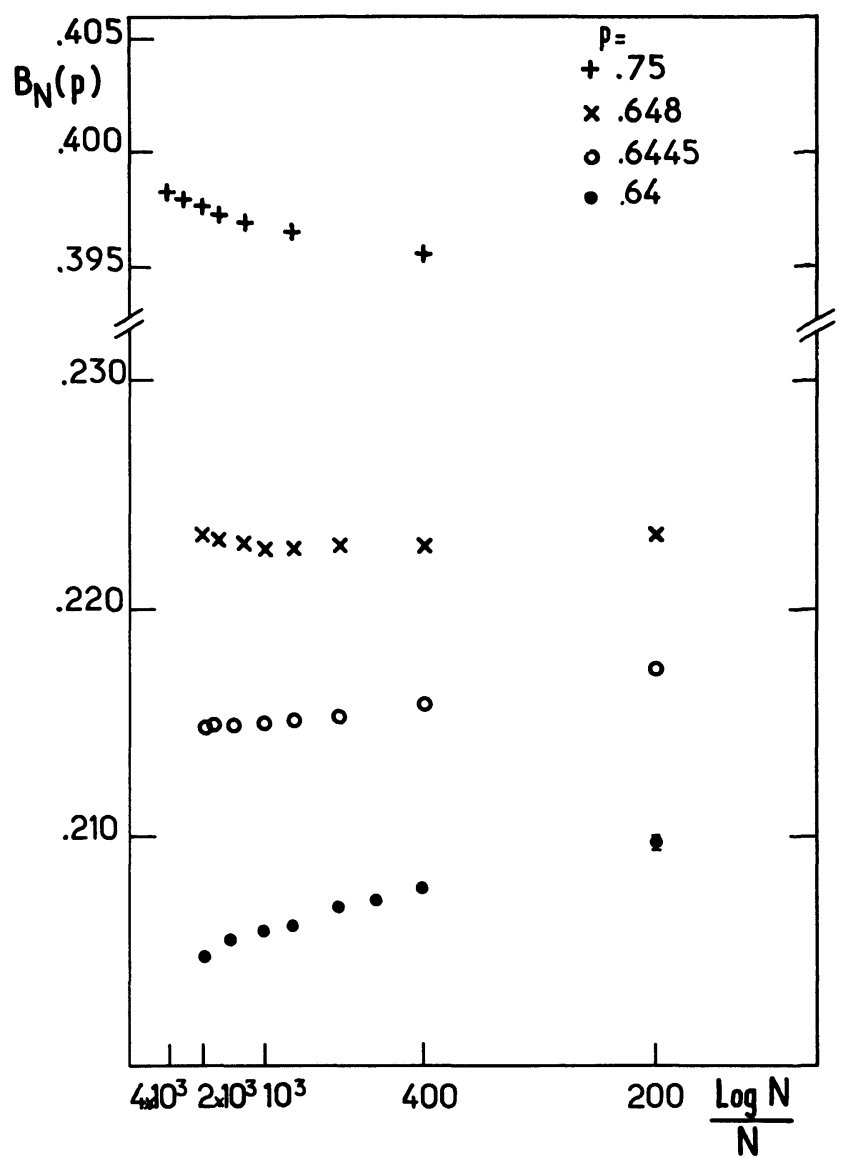

Fig. 7. - Asymptotic behaviour of $B_{N}(p)$ in the bond dilution model, plotted as a function of $\frac{\log N}{N}$, for several values of $p$. The slope measured for $N \geqslant 10^{3}$ (up to $4 \times 10^{3}$ for $p=0.75$ ) gives an effective exponent $\gamma$ that varies with $p$.

a plot of the data of figure 7 versus $N^{-1 / 2}$ gives equally plausible results. A better theoretical understanding of these corrections is required before a reliable analysis can be performed.

\section{Conclusion.}

We have studied a model of Directed Self-Avoiding Walks on a dilute lattice, by various approaches. We have shown that, for any amount of disorder, the most probable and the mean value of the number $\omega_{N}$ of $N$-step walks are different.

More precisely, the distribution of $\omega_{N}$ has two parts : a delta function at $\omega=0$, which corresponds to samples for which there is no SAW of $N$ steps, and a non-trivial part whose weight in the infinite $N$ limit is the probability $P(p)$ that the origin belongs to the infinite cluster. The latter part is peaked around the most probable value $\omega_{N}^{*} \sim \exp \overline{\left(\log \omega_{N}\right)}$, and has a long tail at large values of $\omega_{N}$, which is reflected in the mean value $\bar{\omega}_{N}=(d p)^{N}$. This was checked in 
particular by Monte-Carlo calculations in two dimensions, generating DSAW up to 2000 steps for several values of $p$, and in a few cases up to 4000 steps. We also obtained numerical evidence for either a change in the exponent $\gamma$ for any $p<1$, or at least an asymptotic behaviour different from the pure case.

The model of DSAW thus appears to shed some light on the controversial problem of SAW in random media, and should provide a useful ground for further study of that intriguing subject.

\section{Acknowledgments.}

It is a pleasure to thank B. Derrida for many discussions, ideas and suggestions during the course of this work and G. Berthaud for his help in programming and using the computer system.

\section{References}

[1] Harris, A. B., Z. Phys. B 49 (1983) 347.

[2] KIM, Y., J. Phys. C 16 (1983) 1345.

[3] See also, on the very related problem of absorption of a SAW by traps, Tanaka, F., J. Phys. A 16 (1983) L 489 ;

Vicsek, T., Kertesz, J., Cserti, J., J. Phys. A 15 (1982) L 189.

[4] Kremer, K., Z. Phys. B 45 (1981) 149.

[5] Lyklema, J. W., Kremer, K., Z. Phys. B 55 (1984)41.

[6] Rammal, R., Toulouse, G., Vannimenus, J., J. Physique 45 (1984) 389.

[7] Chakrabarti, B. K., Kertesz, J., Z. Phys. B 44 (1981) 221.

[8] Harris, A. B., J. Phys. C 7 (1974) 1671.

[9] Derrida, B., J. Phys. A 15 (1982) L 119.

[10] Derrida, B., Phys. Rep. 103 (1984) 29.

[11] Hiley, B. J., Finney, J. L., Turke, T., J. Phys. A 10 (1977) 197.

[12] Roy, A. K., Chakrabarti, B. K., Z. Phys. B 55 (1984) 131.

[13] McKenzie, D. S., Phys. Rep. 27C (1976) 35.

[14] Chakrabarti, B. K., Manna, S. S., J. Phys. A 16 (1983) L 113 ;
Blöte, H. W. J., Hilhorst, H. J., J. Phys. A 16 (1983) 3687

Zhang, Z., Yang, Y. S., Yang, Z. R., J. Phys. A 17 (1984) 245 ;

Redner, S., Majid, I., J. Phys. A 16 (1983) L 307 ;

CARdy, J. L., J. Phys. A 16 (1983) L 355 ;

SzPILKA, A. M., J. Phys. A 16 (1983) 2883 ;

SHAPIR, Y., preprint.

[15] Note that these considerations hold also for nondirected systems. For example, if one considers the distribution for the number of SAW of $N$ steps, the quantity analogous to $P_{N}$ will be equal to the probability that $S_{N}$ is not zero, $S_{N}$ being the number of sites that are accessible in exactly $N$ steps from 0 , see Ref. [16].

[16] Vannimenus, J., Nadal, J. P., Martin, H., J. Phys. $A 17$ (1984) L 351.

[17] Dhar, D., Barma, M., J. Phys. C 14 (1981) L 1 ; DE'Bell, K., Essam, J. W., J. Phys. A 16 (1983) 3145

[18] KinzeL, W., in Percolation structures and processes, Eds. G. Deutscher, R. Zallen, J. Adler (Bristol, Adam Hilger) 1982. 